\title{
Survivin in Cancer: A Spider in the Web
}

\section{Sishir K Kamalapuram, Rupinder K Kanwar and Jagat R Kanwar*}

Nanomedicine- Laboratory of Immunology \& Molecular Biomedical Research (LIMBR), Centre for Biotechnology and Interdisciplinary Biosciences, Institute for Frontier Materials (IFM), Deakin University, Australia

It has not been attributed to the ignitable curiosity of the cancer researchers in propagating survivin-ism, it would have certainly be an impossibility to dissect the multifaceted role of survivin in tumour biology [1]. The predominant factorability of survivin in modulating cancer is envisaged in the realms of diagnostic and prognostic abilities, preferential modulation of intricate evolving cancer cell and stem cell signalling paradigms and pharmacological retaliation against prevailing therapeutic efficacies. Therefore, this column provides a snapshot of recent research and development pertaining to survivin biology in cancer $[1,2]$.

\section{Diagnostic and Prognostic Strategies}

Multiplexed nanoflare devices comprising of gold nanoparticle conjugates hybridised with the reporter, quencher and flourophore molecules, had enabled to efficiently detect survivin messenger RNA (mRNA) at relative very low concentrations [3]. Combinatorial multimodal nanotheranostic agents comprising of silencing RNA (siRNA) conjugates, membrane translocation peptides and flourophores coupled to the magnetic nanoparticles, have concomitantly achieved survivin abrogation and therapeutic imaging by magnetic resonance imaging and fluorosenesce imaging [4]. Detection of exosomes with survivin as a cargo protein has invigorated a relative novel speculation, pertaining to the role of survivin in angiogenic propagation with excessive stabilisation and secretion of exosomes among the cancer cells [5]. Furthermore, in addition to the already existing debate about the dichotomic role of nuclear and cytoplasmic survivin, nuclear survivin was detected proportionately along with Phosphohistone- $\mathrm{H} 3$ ( $\mathrm{pHH} 3$ ), prominent mitotic count biomarker [6]. Nevertheless, survivin is attributed in promoting drug resistant tumours. In accordance with the previous observations, nuclear and cytoplasmic counterparts showed a predominant role in stabilisation of drug resistance in cervical carcinoma, in response to the radiation therapy [7].

\section{Cancer Cell Signalling Paradigms}

Critical role of survivin in transcriptional inhibition of $\mathrm{p} 21$ gene, a crucial associate with $\mathrm{p} 53$ tumour suppression gene via p53 signalling mechanisms was noted [8]. Further, novel orphan nuclear receptor TR3 (NR41A) overexpression in lung carcinoma subjects, was promoted by survivin via 553 and mammalian target of rapamycin (mTOR) dependent fashion [9]. Furthermore, systemic modulation of the cell signalling mechanisms including sphingosine-1-phosphate receptor 1 ( a G-protein-coupled receptor for lysophospholipid sphingosine1-phosphate (S1P)) - Signal transducer and activator of transcription 3 (S1PR1-STAT3) signalling [10], modulation of DNA methylatory mechanisms [11], Yes-associated protein (YAP) modulation in conjucture with epidermal growth factor receptor (EGFR), and hippopotamus like phentotype tumour growth - Yes-associated protein (hippo - YAP) organogenesis developmental pathway [12], modulation of an array of microRNA's (miR-542-3p, miR-494, miR-320a, miR-218, miR-708 and miR-203) [13], intervention with tumour necrosis factor apoptosis inducing ligand (TRAIL) associated anticancer therapies [14], modulation of mitochondrial apoptosis in association with second mitochondrial-derived activator of caspase (Smac) in receptor activating protein 1 (RIP1)/caspase-8, and Nuclear Factor-Kappa Beta (NF-kB) signalling was noted [15].

\section{Cancer Stem Cell Signalling Paradigms}

Survivin was significantly associated with the novel stem cell signalling mechanisms, including cluster of differentiation $34^{+}$ $\left(\mathrm{CD} 34^{+}\right)$and differentiation 38 (CD38) leukemic stem/progenitor cell mechanisms associated with unfavourable clinical prognosis [16], regulation of human telomerase reverse transcriptase and survivin specific Cytotoxic T Lymphocytes (CTLs) signaling [17], survivin-DEx3, a survivin splice variant based modulation of cell cycle check point (Chk2) phosphorylation leading DNA damage promoting resistance to genotoxic therapies [18], modulation of sonic hedgehog (SHh) developmental signalling pathway to promoted epithelial mesenchymal transition in promoting pancreatic cancer stem growth [19], modulation of transforming growth factor (TGF)- $\beta$ signalling in hepatic cancer cells, thereby affecting Histone Acetylation Transferases (HATs) and Histone Deacetylases (HDACs) mechanisms that promote chromatin remodeling, and fluxation of antiapoptotic products in hepatic stem cell growth [20]. Pertaining to immunological aspects, survivin was known to critically modulate Signal transducer and activator of transcription (STAT) signalling cascades, having a regulatory effect on MyeloidDerived Suppressor Cell (MDSC) promoting stem cell progression in tumour environment [21].

\section{Therapeutic Strategies Targeting Survivin}

Novel nanoscale drug formulations have been formulated including bioreducible poly (b-amino esters) (PAEs), poly[bis(2hydroxylethyl)-disulfide-diacrylate-b-tetraethylenepentamine] (PAP) nanoformulation, for the delivery of short hair pin RNA (shRNA) and siRNA for multidrug resistant protein (MDR) - P - glycoprotein protein (PgP)) and survivin - iMdr-1-shRNA and iSurvivinshRNA in doxorubicin resistant human breast cancer cells (MCF7) [22], combinatorial formulation of b-cyclodextrin (b-CyD) and polyethylenimine (PEI) nanomicelle for codelivery of survivin shRNA and paclitaxel in ovarian cancer cells (SKOV-3) [23], fabrication of lipid (DOTAP/DOPE) and apolipoprotein (APOA-I) - cytochrome $\mathrm{C}$ for intracellular targeted drug delivery in in vivo lung cancer cell

*Corresponding author: Jagat R Kanwar, Nanomedicine- Laboratory of Immunology \& Molecular Biomedical Research (LIMBR), Centre for Biotechnology and Interdisciplinary Biosciences, Institute for Frontier Materials (IFM), Deakin University, Geelong, Victoria 3217, Australia, Tel: 0061-3-5227 1148; Fax: 0061-3-5227 2539; E-mail: jagat.kanwar@deakin.edu.au

Received September 20, 2012; Accepted September 20, 2012; Published September 21, 2012

Citation: Kamalapuram SK, Kanwar RK, Kanwar JR (2012) Survivin in Cancer: A Spider in the Web. J Nanomed Nanotechol 3:e118. doi:10.4172/2157$7439.1000 \mathrm{e} 118$

Copyright: (c) 2012 Kamalapuram SK, et al. This is an open-access article distributed under the terms of the Creative Commons Attribution License, which permits unrestricted use, distribution, and reproduction in any medium, provided the original author and source are credited. 
model (NCI-H460) [24], multifunctional HER2 monoclonal antibody conjugated RNase A-associated CdTe quantum dot cluster (HER2RQDs) nanoprobe, for therapeutic imaging and targeted delivery in in vivo gastric cancer cell model (MCG803) [25], Magnetic resonance (MR) sensitive liposomal nanoformulation entrapped siRNA (LEsiRNA) specific to survivin, promoted enhanced down regulation of survivin in vivo, leading to remarkable tumour reduction [26].

\section{Future Directions and Challenges of Survivin-ism: Where do we go?}

Needless to say, there is an incessant necessity to fabricate ecofriendly natural product derived multifunctional nanotheranostics that can offer lucrative advantages and ulterior therapeutic possibilities, in comparison with conventional drugs [4]. Novel anticancer nanotherapeutics devised at our laboratory yielded remarkable in vivo anticancer efficacy, in both breast and colon cancer models, parameterized by restoration of a myriad of critical apoptotic signalling cascades [27]. Furthermore, from a futuristic point of view, synergism of the evolving research platforms such as cancer systems biology, bioinformatics, computational chemistry, molecular nanotechnology, quantum medicine, enables the scientific community to concretely understand the evolving cancer growth mechanisms and also designing of drugs at atomic precise, to aptly target the spider - survivin in the web of cancer signalling mechanisms [2].

\section{References}

1. Holmes D (2012) Cancer drug's survivin suppression called into question. Nat Med 18: 842-843.

2. Kanwar JR, Kamalapuram SK, Kanwar RK (2012) Survivin signaling in clinical Oncology: A Multifaceted Dragon. Med Res Rev.

3. Prigodich AE, Randeria PS, Briley WE, Kim NJ, Daniel WL, et al. (2012) Multiplexed nanoflares: mRNA detection in live cells. Anal Chem 84: 2062 2066.

4. Mura S, Couvreur P (2012) Nanotheranostics for personalized medicine. Adv Drug Deliv Rev.

5. Kharaziha P, Ceder S, Li Q, Panaretakis T (2012) Tumor cell-derived exosomes: A message in a bottle. Biochim Biophys Acta 1826: 103-111.

6. Brunner A, Riss P, Heinze G, Brustmann H (2012) pHH3 and survivin are coexpressed in high-risk endometrial cancer and are prognostic relevant. $\mathrm{Br} J$ Cancer 107: 84-90.

7. Chaopotong P, Kajita S, Hashimura M, Saegusa M (2012) Nuclear survivin is associated with cell proliferative advantage in uterine cervical carcinomas during radiation therapy. J Clin Pathol 65: 424-430.

8. Tang L, Ling X, Liu W, Das GM, Li F (2012) Transcriptional inhibition of p21WAF1/CIP1 gene (CDKN1) expression by survivin is at least partially p53dependent: evidence for survivin acting as a transcription factor or co-factor. Biochem Biophys Res Commun 421: 249-254.

9. Lee SO, Andey T, Jin UH, Kim K, Singh M, et al. (2012) The nuclear receptor TR3 regulates $\mathrm{mTORC} 1$ signaling in lung cancer cells expressing wild-type p53. Oncogene 31: 3265-3276.

10. Deng J, Liu Y, Lee H, Herrmann A, Zhang W, et al. (2012) S1PR1-STAT3 signaling is crucial for myeloid cell colonization at future metastatic sites. Cancer Cell 21: 642-654.

11. De Carvalho DD, Sharma S, You JS, Su SF, Taberlay PC, et al. (2012) DNA methylation screening identifies driver epigenetic events of cancer cell survival. Cancer Cell 21: 655-667

12. Huang JM, Nagatomo I, Suzuki E, Mizuno T, Kumagai T, et al. (2012) YAP modifies cancer cell sensitivity to EGFR and survivin inhibitors and is negatively regulated by the non-receptor type protein tyrosine phosphatase 14 . Oncogene.

13. Xie Y, Tobin LA, Camps J, Wangsa D, Yang J, et al. (2012) MicroRNA-24 regulates XIAP to reduce the apoptosis threshold in cancer cells. Oncogene.
14. Dimberg LY, Anderson CK, Camidge R, Behbakht K, Thorburn A, et al. (2012) On the TRAIL to successful cancer therapy? Predicting and counteracting resistance against TRAIL-based therapeutics. Oncogene.

15. Wagner L, Marschall V, Karl S, Cristofanon S, Zobel K, et al. (2012) Smac mimetic sensitizes glioblastoma cells to Temozolomide-induced apoptosis in a RIP1- and NF-kB-dependent manner. Oncogene.

16. Carter BZ, Qiu Y, Huang X, Diao L, Zhang N, et al. (2012) Survivin is highly expressed in CD34(+)38(-) leukemic stem/progenitor cells and predicts poor clinical outcomes in AML. Blood 120: 173-180.

17. Anguille S, Van Tendeloo VF, Berneman ZN (2012) Leukemia-associated antigens and their relevance to the immunotherapy of acute myeloid leukemia. Leukemia.

18. Lopergolo A, Tavecchio M, Lisanti S, Ghosh JC, Dohi T, et al. (2012) Chk2 phosphorylation of survivin-DeltaEx3 contributes to a DNA damage-sensing checkpoint in cancer. Cancer Res 72: 3251-3259.

19. Tang SN, Fu J, Nall D, Rodova M, Shankar S, et al. (2012) Inhibition of sonic hedgehog pathway and pluripotency maintaining factors regulate human pancreatic cancer stem cell characteristics. Int J Cancer 131: 30-40.

20. Majumdar A, Curley SA, Wu X, Brown P, Hwang JP, et al. (2012) Hepatic stem cells and transforming growth factor $\beta$ in hepatocellular carcinoma. Nat Rev Gastroenterol Hepatol 9: 530-538.

21. Gabrilovich DI, Ostrand-Rosenberg S, Bronte V (2012) Coordinated regulation of myeloid cells by tumours. Nat Rev Immunol 12: 253-268.

22. Yin Q, Shen J, Chen L, Zhang Z, Gu W, et al. (2012) Overcoming multidrug resistance by co-delivery of Mdr-1 and survivin-targeting RNA with reductionresponsible cationic poly( $\beta$-amino esters). Biomaterials 33: 6495-6506.

23. Hu Q, Li W, Hu X, Hu Q, Shen J, et al. (2012) Synergistic treatment of ovarian cancer by co-delivery of survivin shRNA and paclitaxel via supramolecular micellar assembly. Biomaterials 33: 6580-6591.

24. Kim SK, Foote MB, Huang L (2012) The targeted intracellular delivery of cytochrome $\mathrm{C}$ protein to tumors using lipid-apolipoprotein nanoparticles. Biomaterials 33: 3959-3966.

25. Ruan J, Song H, Qian Q, Li C, Wang K, et al. (2012) HER2 monoclonal antibody conjugated RNase-A-associated CdTe quantum dots for targeted imaging and therapy of gastric cancer. Biomaterials 33: 7093-7102.

26. Kesharwani P, Gajbhiye V, Jain NK (2012) A review of nanocarriers for the delivery of small interfering RNA. Biomaterials 33: 7138-7150.

27. Kanwar JR, Mahidhara G, Kanwar RK (2012) Novel alginate-enclosed chitosancalcium phosphate-loaded iron-saturated bovine lactoferrin nanocarriers for oral delivery in colon cancer therapy. Nanomedicine (Lond). 